\title{
Limitations in the efficiency of fluorescent whitening agents in uncoated paper
}

\author{
Ludovic G. Coppel, Mattias Andersson, Per Edström and Jussi Kinnunen
}

\begin{abstract}
KEYWORDS: Whiteness, Kubelka-Munk, Greening, Fluorescence, Fluorescent whitening agents

SUMMARY: The fluorescence efficiency of one fluorescent whitening agent (FWA) in uncoated and unfilled paper is characterised at different FWA concentrations. An extended Kubelka-Munk model proposed earlier by several authors is applied to quantify the effect on CIE whiteness of light absorption by the FWA in the visible spectrum. At high FWA concentration, chemical interactions slightly modify the quantum efficiency, which then depends on the FWA concentration. This effect has however a negligible effect on the CIE whiteness for FWA concentrations used in practice. Light absorption by the FWA in the visible spectrum is shown to be the main cause of greening (a shift of the chromaticity towards green) and saturation of the fluorescence effect. With increasing FWA concentration, the positive effect of fluorescence is neutralised by the reduction of the reflected radiance factor in the violet-blue region of the spectrum induced by a significant absorption by the FWA in that region. The effect is well predicted with a simple extension of the Kubelka-Munk model for light scattering in paper.
\end{abstract}

\section{ADDRESSES OF THE AUTHORS: Ludovic Gustafsson Coppel (ludovic.coppel@innventia.com), Innventia AB, Box 5604, SE-114 86 Stockholm. \\ Mattias Andersson (mattias.andersson@miun.se), Digital Printing Center, Mid Sweden University, SE-891 18 Örnsköldsvik, Sweden. Per Edström (per.edstrom@miun.se), Mid Sweden University, Department of Natural Sciences, Engineering and Mathematics, SE-871 88 Härnösand, Sweden. Jussi Kinnunen (jussi.kinnunen@uef.fi), University of Eastern Finland, Department of Physics and Mathematics, P.O. Box 111, FI-80101 Joensuu, Finland. Corresponding author: Mattias Andersson}

Whiteness is an important quality parameter of paper and a marketing goal for product segments such as office papers. This has led to more bleaching and a substantial increase in the concentration of fluorescent whitening agents (FWA) added in paper. FWA are dyes that absorb ultraviolet (UV) radiation and emit in the blue region of the spectrum, hence increasing the perceived whiteness by both increasing the lightness and the blueness of the paper. Among the numerous whiteness formulae proposed over the years, the CIE whiteness formula (ISO 11475) is the most widely used. CIE whiteness has been found to correlate well with visual estimation for many white samples having similar tint or fluorescence (Uchida
1998). Ganz and Pauli (1995) derived an approximation of the CIE whiteness based on the $L^{*} a^{*} b^{*}$ colour space. Paper is whiter when $L^{*}$ is large and $b^{*}$ shows a large negative value, i.e. high luminance and bluish tint.

An increase in the FWA concentration initially leads to a substantial whiteness increase but the whiteness tends to saturate at higher FWA concentration (Allen 1964). With further FWA addition, the whiteness can even decrease and the paper turns greenish. This "greening effect" is well known in the industry but little has been published, except for industrial White Papers (Bayer 2003; Roick, Hunke 2003; Puebla 2006). This effect is more problematic in coatings, but whiteness saturation in plain paper is also related to a change of $a^{*}$ towards green. Shakespeare and Shakespeare (1999) attributed the greening effect to a red-ward shift of the fluoresced light with increasing FWA concentration, to quantum yield reduction, and to absorption in the violet-blue region when FWA molecules aggregate. However, to the authors' knowledge, these three contributions have never been quantified individually.

Better understanding of the dependence of the fluorescence on FWA concentration is obtained by separating the fluorescence mechanisms intrinsic to the FWA from the scattering and absorption of the furnish. This in turn requires modelling the light scattering process in a fluorescing paper. Light scattering in paper is most frequently modelled with the Kubelka-Munk $(1931 ; 1948)$ theory (K-M). The $\mathrm{K}-\mathrm{M}$ theory relates two reflectance factor measurements to a scattering coefficient, $S$, and an absorption coefficient, $K$. Several extensions of the K-M theory have been developed over the years to account for fluorescence (Allen 1964; Fukshansky, Kazarinova 1980; Bonham 1986; Shakespeare 2000). However, these extensions require either a defined polychromatic illumination or a semiinfinite layer. More recently, Kokhanovsky (2009; 2010) presented a general analytical solution. In all extended K-M models, fluorescence is modelled by letting part of the light absorbed at an excitation wavelength $\mu$ be re-emitted at a longer wave-length $\lambda$, according to a dimensionless spectral quantum yield, or quantum efficiency $Q(\mu, \lambda)$, which determines the intrinsic emission spectrum of the FWA (see Appendix 1 for the notations used in this paper). 


\section{Measuring fluorescence in paper}

The paper industry measures whiteness and fluorescence with a standardised instrument (ISO 2469) using a diffuse illumination and a detector at the normal angle to the paper surface. This geometry is referred to as the $\mathrm{d} / 0^{\circ}$ geometry. A d $0^{\circ}$ instrument is equipped with a broad band light source and a UV-adjustment filter controlling the relative UV-content in the illumination. The instrument measures the total radiance factor, $\beta_{\mathrm{T}}(\lambda \mid E)$, at each wavelength $\lambda$ for a given illumination $E$. When the sample does not fluoresce, the total radiance factor is independent of the illumination and the spectral distribution of the light source is not of concern. However, for fluorescing samples, the measured total radiance is the sum of the reflected radiance factor, $\beta_{\mathrm{R}}(\lambda)$, and the luminescent radiance factor, $\beta_{\mathrm{L}}(\lambda \mid \mathrm{E})$. The luminescent radiance factor depends on the spectral distribution of the light source. In $\mathrm{d} / 0^{\circ}$ instruments, the UVcontent is adjusted to measure the total radiance factor for a given illuminant. This adjustment follows a standardised procedure involving a hierarchy of standardizing and authorised laboratories. The CIE whiteness is standardised for two illuminants, D65 with a $10^{\circ}$ standard observer (ISO 11475), and $\mathrm{C}$ with a $2^{\circ}$ standard observer (ISO 11476). The calibration of the instrument is pragmatic and a compromise between different factors with the primary goal to ensure the greatest possible statistical reproducibility of the measurements. The calibration of $\mathrm{d} / 0^{\circ}$ instruments is specific for the type of FWAs used in paper and is in some respects arbitrary (CIE 182, 2007). Moreover, the use of a polychromatic illumination does not allow a correct determination of the quantum efficiency.

An accurate determination of the total radiance factor and quantum efficiency requires the use of a two-monochromator instrument, or bispectrometer. With this instrument it is possible to record the spectral radiance of the sample for each illumination wavelength. This results in a two-dimensional matrix of bispectral radiance factor data, $B(\mu, \lambda)$, known as the Donaldson matrix (Donaldson 1954). The diagonal elements of the Donaldson matrix, for which excitation wavelength $\lambda$ and emitted wavelength $\mu$ are equal, is the reflected radiance factor $\beta_{\mathrm{R}}(\lambda)$. From the Donaldson matrix, it is possible to accurately calculate the total radiance factor $\beta_{\mathrm{T}}(\lambda \mid E)$ for any spectral distribution of the illumination.

\section{FWA's absorption band and quantum efficiency}

FWAs absorb UV radiation and short wavelength visible light, at wavelengths ranging from $240 \mathrm{~nm}$ to
$420 \mathrm{~nm}$, and emit in the violet-blue, mainly between $380 \mathrm{~nm}$ to $500 \mathrm{~nm}$ (Shakespeare 2000). This means that the absorption and emission bands of FWAs overlap and that FWAs absorb not only UV radiation but also some violet-blue light in the visible spectrum. An increase in the FWA concentration will thus lead to a decrease in the reflected radiance factor in that region of the spectrum.

The fluorescence efficiency will depend on the amount of radiation reaching the FWA molecules, and on the scattering and absorption properties of the substrate at the emitted wavelengths. Measured radiance factors or bispectral radiance factors are thus not a direct measure of the intrinsic light (or radiation) absorption and light emission properties of the FWA. The radiance factor will for instance depend on the absorption of the substrate in the blue region of the spectrum, while the quantum efficiency $Q(\mu, \lambda)$ relates the amount of photons emitted from the FWA at wavelength $\lambda$ to the amount of photons absorbed by the FWA at wavelength $\mu$.

The calibration routine of the $\mathrm{d} / 0^{\circ}$ instrument described above relies on the fact that the shape of the quantum efficiency is nearly independent of the excitation wavelength (Allen 1957; Bristow 1994). On the other hand, Shakespeare (2000) observed a red-ward shift of the quantum efficiency with increasing FWA concentration.

\section{Aim of the present study}

The purpose of the present work is to test whether absorption by the FWA in the visible spectrum and concentration-dependent quantum efficiency can explain whiteness saturation and greening in uncoated papers. An extended K-M model including fluorescence is used to determine the model parameters $S, K$, and $Q$ at different FWA concentrations. Simulations of hypothetical samples of constant quantum efficiency with concentration, and with zero absorption in the visible spectrum, are then carried out. By comparing these ideal samples to the real samples, concentration-dependent quantum efficiency and absorption by the FWA in the visible spectrum are studied separately.

\section{Materials and Methods}

A set of uncoated paper samples with increasing FWA concentration, but otherwise identical, is produced. A bispectrometer is used to obtain the Donaldson matrix and compute the total radiance factor $\beta_{\mathrm{T}}(\lambda \mid \mathrm{D} 65)$ for a D65 illuminant. An extended $\mathrm{K}-\mathrm{M}$ model is used to determine the model parameters $S(\lambda), K(\lambda)$, and $Q(\mu, \lambda)$ at each FWA concentration. The CIE $L^{*} a^{*} b^{*}$ values are 
computed from $\beta_{\mathrm{T}}(\lambda \mid \mathrm{D} 65)$ (Wyszecki, Stiles 2000). The CIE whiteness equation is then used to plot the whiteness as function of the FWA concentration.

To quantify the effect of a change in the quantum efficiency with increasing FWA concentration, a set of samples with constant $Q$ should be compared to a similar set with $Q$ varying with FWA concentration. Since it is not possible to produce such real samples, simulations by means of the extended K-M model are used instead. Having determined $Q$ at one FWA concentration, the same $Q$ is used for all other FWA concentrations. The resulting whiteness, $W_{\mathrm{CIE}}$, and the $a^{*}$ value are then compared to those obtained for the real samples. In a similar manner, the effect of light absorption by the FWA in the visible spectrum is quantified by comparing simulated samples, with no light absorption by the FWA in the visible spectrum, to the real samples.

The increase in whiteness with FWA concentration will depend on the ability of the furnish to adsorb FWA. The actual FWA concentration in the dried samples is not known and may not be linear with the amount of FWA fed into the pulp. Hence, whiteness saturation may occur due to retention problems. The purpose here is thus not to predict whiteness from fed FWA concentration, but to isolate two additional effects to whiteness saturation. The simulations are therefore computed from the estimated (measured) absorption coefficient at each FWA concentration. In order to relate the studied effects to the actual FWA concentrations used, whiteness is nonetheless plotted as function of the FWA concentration in the pulp.

In order to relate our results to industrial whiteness measurement made with (single-monochromator) $\mathrm{d} / 0^{\circ}$ instruments, the $L^{*} a^{*} b^{*}$ and whiteness values measured with a $\mathrm{d} / 0^{\circ}$ spectrophotometer are first compared to those obtained with the $0^{\circ} / 45^{\circ}$ bispectrometer.

\section{Production of paper samples}

Paper samples with a final basis weight of $80 \mathrm{~g} / \mathrm{m}^{2}$ were produced on the experimental pilot paper machine XPM at MoRe Research, Örnsköldsvik, Sweden. The samples were formed from a pulp blend consisting of $75 \%$ hardwood and $25 \%$ softwood. The samples were sized using a mix of Kemsize $(0.04 \%)$ and $C^{*}$ bond starch $(0.06 \%)$ in order to form samples with a target Cobb-value of 25 (varied between 25 and 28). Percol (0.01\%) and Bentonit $(0.05 \%)$ were used as retention agents. No fillers or shading dyes were used. The samples were neither surface sized nor calendered. A tetrasulpho FWA, Blankophor P01, was added to the pulp at 10 different concentrations $(0,0.75,1.5,3,6,9,12,15$, 18 , and $36 \mathrm{~kg} / \mathrm{T})$. The $\mathrm{D} 65 / 10^{\circ} \mathrm{CIE}$ whiteness ranges from 73, for the undyed sample, to 142 for the sample with $36 \mathrm{~kg} / \mathrm{T}$ FWA. The ISO brightness of the undyed sample is 87 .

\section{Optical characterisation of the samples}

The samples were measured with a Technidyne ColourTouch $\mathrm{d} / 0^{\circ}$ spectrophotometer calibrated in accordance with ISO 2469. The UV amount was adjusted to $\mathrm{UV}(\mathrm{D} 65)$ and $\mathrm{UV}(\mathrm{C})$ conditions in accordance with ISO 11475 and ISO 11476 respectively. The spectrophotometer operates at a wavelength range between $380 \mathrm{~nm}$ and $740 \mathrm{~nm}$ with $10 \mathrm{~nm}$ intervals.

The Donaldson matrix was obtained with a custom-made bispectrometer (Turunen et al., 2010) at the Infotonics Centre, University of Eastern Finland in Joensuu. The bispectrometer is assembled on an optical table. It consists of a water cooled $1 \mathrm{~kW}$ Xenon light source (Oriel M-66923 housing, Oriel 6271 Xe OF lamp, Oriel 6123 filter, Newport corporation, Irvine, California), a CzernyTurner monochromator (GM 252, Schoeffel Instruments Div., Kratos Inc., Westwood, New Jersey), a Glan Taylor polarizer (GT10, Thorlabs, Newton, New Jersey) and spectrograph detector (PMA-11, Hamamatsu Photonics K.K., Japan) that operates at a wavelength range between 200 and $950 \mathrm{~nm}$ with $2 \mathrm{~nm}$ intervals wavelength resolution. Each measurement used two perpendicular linear polarisation angles, $0^{\circ}$ and $90^{\circ}$, that were averaged after Donaldson matrix calculations.

A moving table allows three samples and a calibration reference sample to be measured sequentially. At each excitation wavelength, the reference plate (Spectralon SRS-99, Labsphere, Inc., North Sutton, New Hampshire) is measured prior to the three samples to correct for variations in the illumination.

The excitation wavelengths used ranged from $280 \mathrm{~nm}$ to $700 \mathrm{~nm}$ with $5 \mathrm{~nm}$ sampling interval and $5 \mathrm{~nm}$ peak half-width. Due to limited time available with the instrument, some samples were measured only up to $540 \mathrm{~nm}$, and some others only up to $600 \mathrm{~nm}$. The measurements were carried out both on single sheets with black background and on opaque pads of identical sheets. The only exception was for the undyed sample, which was only measured for the case of an opaque pad. The radiance factor can be computed for any illuminant from the Donaldson matrix by weighting the bispectral radiance factor with the normalised spectral distribution of the illuminant $E$, and adding it to the reflected radiance factor (Zwinkels, Gauthier, 1999):

$$
\beta(\lambda \mid E)=\beta_{\mathrm{R}}(\lambda)+\frac{1}{E(\lambda)} \sum_{\mu<\lambda} B(\mu, \lambda) E(\mu)
$$




\section{Modelling fluorescence with an extended Kubelka- Munk model}

For a medium of infinite thickness, the Kokhanovsky $(2009 ; 2010)$ model gives the same equation as the one obtained by Shakespeare and Shakespeare (2003) and Allen (1964). The Donaldson matrix is computed from the scattering and absorption coefficients, and the quantum efficiency through

$$
\begin{aligned}
& B(\mu, \lambda>\mu)=\frac{K_{F}(\mu) Q(\mu, \lambda)}{2(M(\mu)+M(\lambda))} \times \\
& \left(2+\frac{K(\mu)}{S(\mu)}-\frac{M(\mu)}{S(\mu)}\right)\left(2+\frac{K(\lambda)}{S(\lambda)}-\frac{M(\lambda)}{S(\lambda)}\right), \\
& B(\mu, \lambda<\mu)=0, \\
& B(\mu, \lambda=\mu)=1+\frac{K(\mu)}{S(\mu)}-\sqrt{\frac{K(\mu)^{2}}{S(\mu)}+2 \frac{K(\mu)}{S(\mu)}},
\end{aligned}
$$

where $M(x)=\sqrt{K(x)^{2}+2 K(x) S(x)}$.

$$
K_{F}(\mu)=K_{\text {dyed }}(\mu)-K_{\text {undyed }}(\mu), \quad \text { introduced by }
$$

Shakespeare and Shakespeare (2003), is the effective absorption coefficient of the fluorescent dye, assuming additivity of the dye and substrate absorption properties. $E q 2$ is originally derived from the assumption that fluorescence is isotropic and that emitted wavelengths are outside the absorption band of the FWA. The latter requires that the emission band of the fluorescent dye does not overlap with its absorption band. However, this only means that the model only allows single fluorescence, i.e. fluoresced light cannot fluoresce further to a longer wavelength. On the other hand, fluoresced light can be absorbed by the fluorescence dye. If the excitation and emission bands of the FWA do not overlap, $K(\lambda)$ can be set to $K_{\text {undyed }}$ in $E q$ 2, since the FWA's absorption is then zero in its emission band. However, if $K(\lambda)>K_{\text {undyed }}$, fluoresced light is absorbed by the FWA, although this does not lead to further fluorescence. The impact of the absorption in the emission band of the FWA is assessed by computing $Q$ with and without this assumption.

Having determined $S$ and $K, Q$ can be determined from the measured $B$ by inverting $E q 2 a$, according to

$$
\begin{aligned}
& Q(\mu, \lambda>\mu)= \\
& \frac{2(M(\mu)+M(\lambda)) B(\mu, \lambda)}{K_{F}(\mu)\left(2+\frac{K(\mu)}{S(\mu)}-\frac{M(\mu)}{S(\mu)}\right)\left(2+\frac{K(\lambda)}{S(\lambda)}-\frac{M(\lambda)}{S(\lambda)}\right)}
\end{aligned}
$$

The scattering coefficient, $S$, and the absorption coefficient, $K$, can be determined in theory with the K-M equations, from measured $\beta_{\mathrm{R}}(\lambda)$ for a single sheet and for an opaque pad of identical sheets. However, this approach is not applicable here due to the fact that the $80 \mathrm{~g} / \mathrm{m}^{2}$ paper samples are opaque in the UV region of the spectrum, already at the lowest FWA concentration. It is therefore assumed here that $S$ is independent of the FWA concentration, allowing its determination from the undyed sample. This assumption is made in contradiction with the findings of Shakespeare and Shakespeare (2003), who showed that $S$ was decreasing with increasing $K$, and hence was dependent on the FWA concentration. On the other hand, $B$ in $E q 2$ is only dependent on the ratio $K(\mu) / S(\mu)$, if $K_{\text {dyed }}(\mu) \gg K_{\text {undyed }}(\mu)$ and $M(\mu)>>$ $M(\lambda)$. Since $S$ decreases with increasing $K, B$ becomes more and more dependent on $K / S$, as $K$ increases. At lower $K$, the decrease in $S$ is limited. Moreover, since $S$ varies rather linearly with the wavelength, it is here extrapolated in the UV region from the spectrophotometer measurements. Having estimated the concentration independent scattering $S$, the absorption coefficient $K$ of the undyed and dyed samples can be obtained from $\beta_{\mathrm{R}}$ at the different FWA concentrations and $Q$ can be determined with the extended K-M model including fluorescence.

The following steps are performed to determine the scattering and absorption coefficients of the samples, and the quantum efficiency $Q$ of the FWA for each sample:

1. $S_{\text {undyed }}$ and $K_{\text {undyed }}$ of the paper without FWA are calculated from the reflected radiance factor of a single undyed sheet on a black background, and the reflected radiance factor of an opaque pad of undyed sheets. Both measurements are made with the spectrophotometer in the wavelength range 380 to $700 \mathrm{~nm}$;

2. $S_{\text {undyed }}$ is extrapolated from $380 \mathrm{~nm}$ down to 280 $\mathrm{nm}$ from a linear fit of $S_{\text {undyed }}$ in the range 400 to $540 \mathrm{~nm}$;

3. The scattering coefficient is assumed to remain constant and independent of FWA addition, $S_{\text {dye }}=S_{\text {undyed }}$ for all samples;

4. $K_{\text {undyed }}$ is recalculated to fit the reflected radiance factor of an opaque pad of undyed sheets measured with the bispectrometer (280-600nm) according to (Kubelka, Munk 1931):

$$
K=\frac{S\left(1-\beta_{R}\right)^{2}}{2 \beta_{R}} \text {. }
$$

The same equation is used to determine the absorption coefficient of the dyed sheets.

The quantum efficiency $Q$ is then calculated with $E q$ 3. Three different hypothetical FWAs are then 
simulated to quantify the effect of a varying $Q$, and the effect of the absorption by the FWA in the visible spectrum, on the CIE $L^{*} a^{*} b^{*}$ and whiteness values. $E q 2$ is used to compute the Donaldson matrix. $E q l$ is then used to compute the total radiance factor using D65 as illuminant. The three hypothetical FWAs are simulated:

1. By using a constant $Q$ for all samples. This simulates a FWA with quantum efficiency independent of the FWA concentration. The sample with $3 \mathrm{~kg} / \mathrm{T}$ FWA is taken as reference point and $Q_{3 \mathrm{~kg} / \mathrm{T}}$ is used for all samples;

2. By using the determined $Q$ for each sample, but setting $K_{\mathrm{F}}(\mu>400 \mathrm{~nm})$ to zero. This simulates the CIE $L^{*} a^{*} b^{*}$ and whiteness values of the samples with a FWA that does not absorb light in the visible part of the spectrum. By comparing the simulated values to the measured values, the effect of the absorption by the FWA on $L^{*} a^{*} b^{*}$ and whiteness can be assessed. However, by setting $K_{\mathrm{F}}(\mu>400 \mathrm{~nm})=0$, the fluorescence at excitation wavelengths above $400 \mathrm{~nm}$ is quenched;

3. By using $Q$ as in 1 and keeping $K_{\mathrm{F}}(\mu)$ unaffected at the excitation wavelength in $E q 2 a$, but setting $K(\lambda)$ at the emitted wavelength equal to $K_{\text {undyed. In }}$ $E q 2 c, K_{\text {undyed }}$ is also used for emission wavelengths above $400 \mathrm{~nm}$. This simulates an ideal FWA with the same total fluorescence as the reference one, but with no absorption in the visible spectrum.

\section{Results}

\section{Measurements}

The radiance factor measured with the spectrophotometer was compared to the radiance factor obtained with the bispectrometer for the $\mathrm{C}$ and D65 illuminants (Fig 1). Despite the differences in measurement geometry, illumination, and calibration procedure, the spectral radiances obtained from the $0^{\circ} / 45^{\circ}$ bispectrometer and the $\mathrm{d} / 0^{\circ}$ spectrophotometer are close. The largest colour difference is $0.6 \Delta \mathrm{E}$ (CIE 1976), for the $6 \mathrm{~kg} / \mathrm{T}$ sample. The largest difference in CIE whiteness (2.5 units) is observed for the same sample. The $L^{*} a^{*} b^{*}$ and whiteness values cannot be compared for the $0.75,1.5$, and $36 \mathrm{~kg} / \mathrm{T}$ samples, since these samples were only measured for a wavelength range from 280 to $540 \mathrm{~nm}$ with the bispectrometer.

The D65/10 $\mathrm{CIE}$ whiteness increases with increasing FWA concentration from 73 for the undyed sample to 141 for the $36 \mathrm{~kg} / \mathrm{T}$ sample (Fig 2, left). When plotting CIE $b^{*}$ values versus FWA concentration, $b^{*}$ initially decreases with increasing FWA concentration down to a saturation point. At the saturation point, both whiteness and $b^{*}$ remain constant although the FWA concentration is increased further. From this point however, the $a^{*}$ value starts to decrease and a greening effect can be observed, Fig 2, right.

\section{Absorption and quantum efficiency}

The calculated absorption and scattering (extrapolated below $400 \mathrm{~nm}$ ) coefficients of the samples are shown in Fig 3. The absorption coefficient is at its maximum at $360 \mathrm{~nm}$. It is compared to the bispectral radiance factor of the 3 $\mathrm{kg} / \mathrm{T}$ sample at this excitation wavelength. For comparison purposes, the bispectral radiance factor is normalised to arbitrary units. The FWA has a significant absorption in the $390-420 \mathrm{~nm}$ range, which overlaps with the FWA's emission band. This overlap occurs for all other excitation wavelengths below $420 \mathrm{~nm}$.

The quantum efficiency of the FWA was determined with $E q 3$ from the obtained Donaldson matrix. The quantum efficiency $Q$ is shown in Fig 4 at $\mu=360 \mathrm{~nm}$ for different FWA concentrations. The total quantum efficiency, $Q_{t o t}(\mu)$, obtained by integrating the spectral quantum efficiency at $\mu=360 \mathrm{~nm}$ is rather constant and the shape of the quantum efficiency of the FWA is similar for all FWA concentrations. However, the quantum efficiency of the FWA is moving towards longer wavelengths (green shift) with increasing FWA concentration.

Since the absorption and emission bands of the FWA overlap, part of the light fluoresced between $400 \mathrm{~nm}$ and $420 \mathrm{~nm}$ is re-absorbed by the FWA (and eventually re-emitted at longer wavelengths). This will influence the determination of the quantum efficiency in the case where it is assumed that the absorption and emission bands of the FWA do not overlap. In Fig 5, the quantum efficiency derived assuming $K(\lambda)=K_{\text {undyed }}$ in $E q 3$ is compared to the quantum efficiency obtained when $K(\lambda)=K_{\text {FWA }}$. The calculated quantum efficiency is lower if this absorption is neglected. This occurs for all excitation wavelengths, although it is only shown here for $360 \mathrm{~nm}$ excitation wavelength. The rather constant quantum efficiency in Fig 4, derived under the assumption that $S$ is independent of the FWA concentration indicates that $Q$ in $E q 2$ is dependent on the ratio $K / S$.

\section{Effect of green shift and blue absorption on whiteness}

In Fig 6, the simulated $a^{*}$ and $b^{*}$ values are compared to those obtained with the bispectrometer. Samples at $0.75,1.5$, and $36 \mathrm{~kg} / \mathrm{T}$ are not measured but only simulated since their Donaldson matrix was 

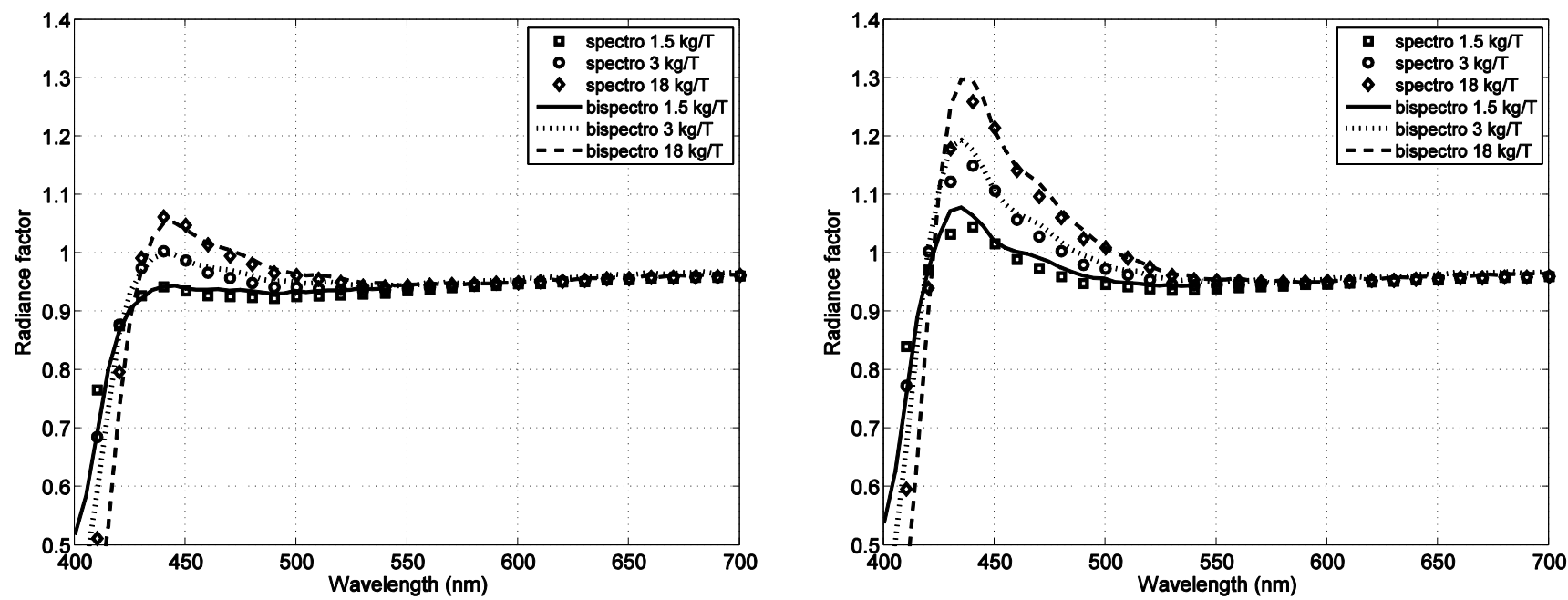

Fig 1. Spectral radiance factor from samples with different FWA concentrations measured with the $d / 0^{\circ}$ ColourTouch spectrophotometer (markers), and computed from the Donaldson matrix measured with the $0^{\circ} / 45^{\circ}$ bispectrometer (lines). Left: C illumination. Right: D65 illumination.
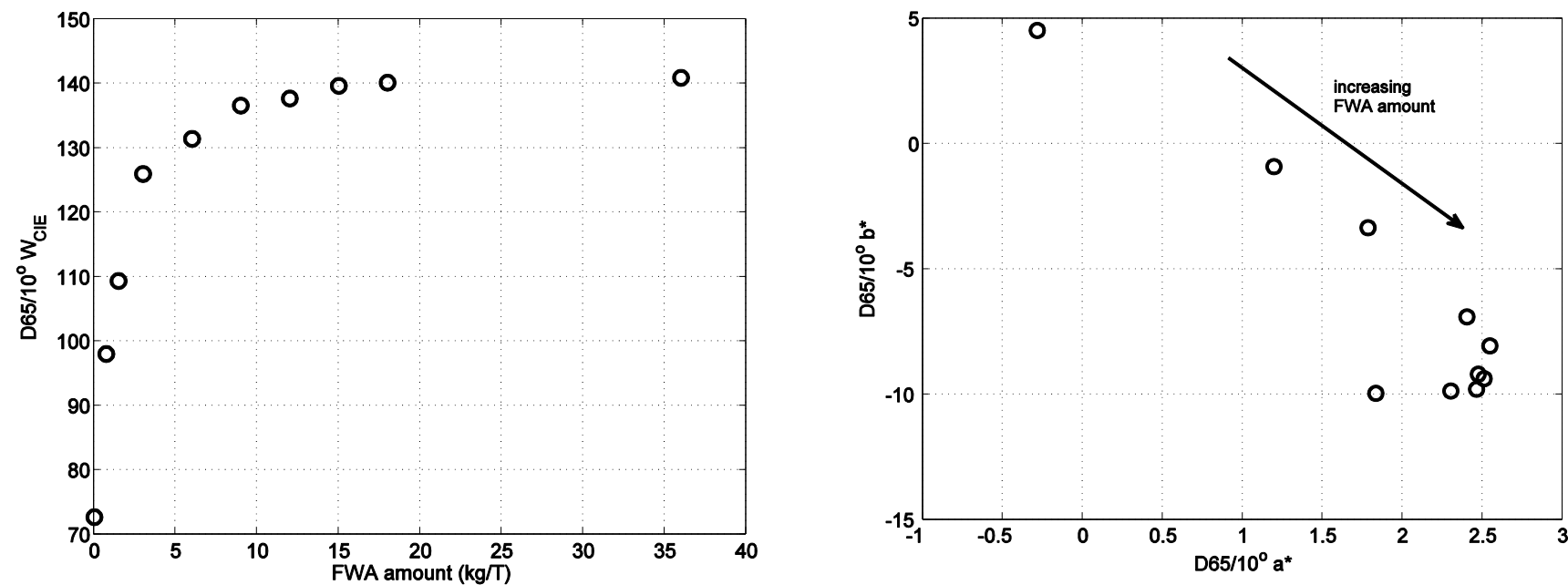

Fig 2. Left: $\mathrm{CIE}$ D65/10 $10^{\circ}$ whiteness measured with spectrophotometer versus FWA concentration. Right: $b^{*}$ versus $a^{*}$. $a^{*}$ starts decreasing at larger FWA concentrations while both $b^{*}$ and $W_{\text {CIE }}$ saturate.

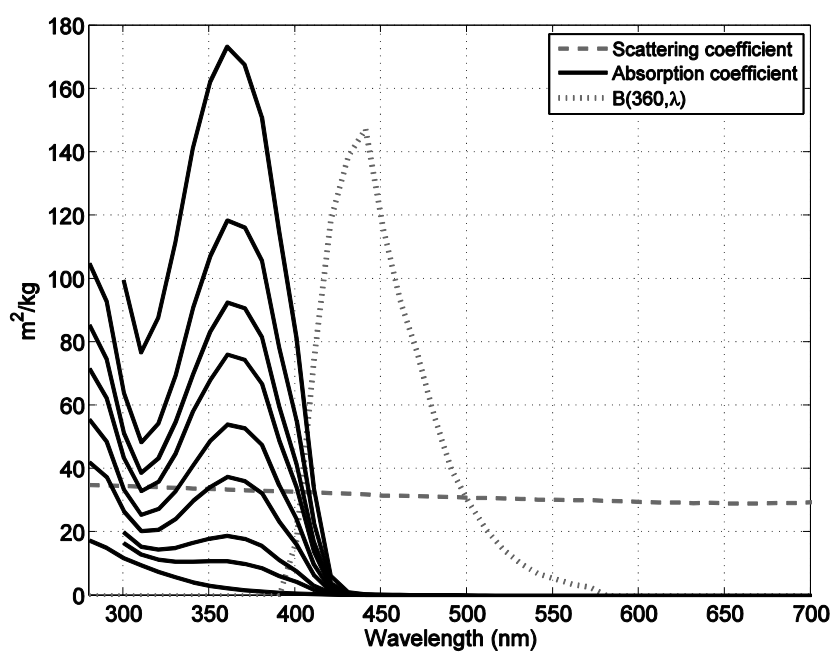

Fig 3. Kubelka-Munk scattering and absorption coefficients of the samples at different FWA concentrations. The bispectral radiance factor of the $3 \mathrm{~kg} / \mathrm{T}$ sample at $360 \mathrm{~nm}$ excitation wavelength shows that the excitation and emission band of the FWA overlap. $B(360, \lambda)$ is here normalised to arbitrary units for comparison.

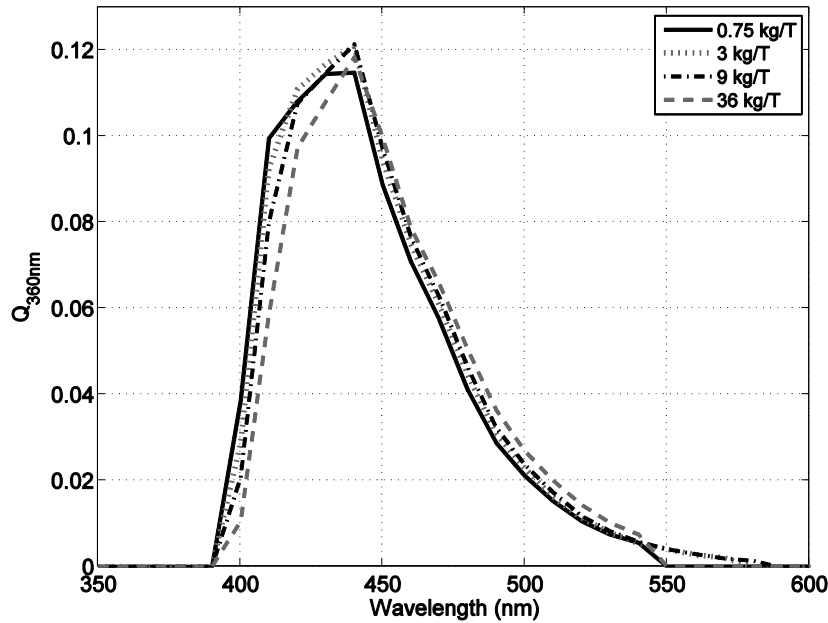

Fig 4. Quantum efficiency at $360 \mathrm{~nm}$ excitation wavelength determined with the extended KM model for different FWA concentrations. The 0.75 and $36 \mathrm{~kg} / \mathrm{T}$ samples were measured only up to $540 \mathrm{~nm}$. 


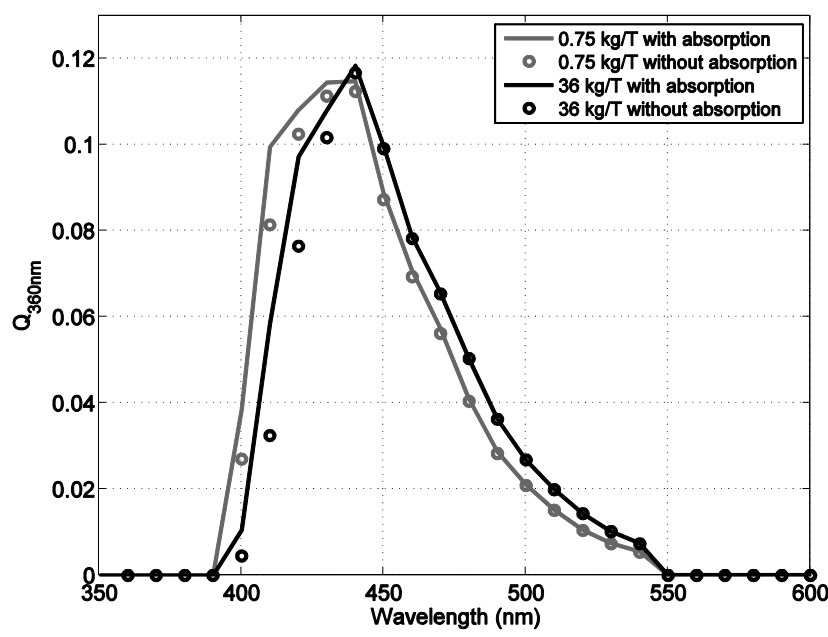

Fig 5. Comparison of the obtained quantum efficiency for cases 1) the FWA does absorb in the emission band; 2) the FWA does not absorb in the emission band. Since fluoresced light in case 2 is not absorbed, the calculated quantum efficiency is lower to account for the observed decrease of the bispectral radiance factor.

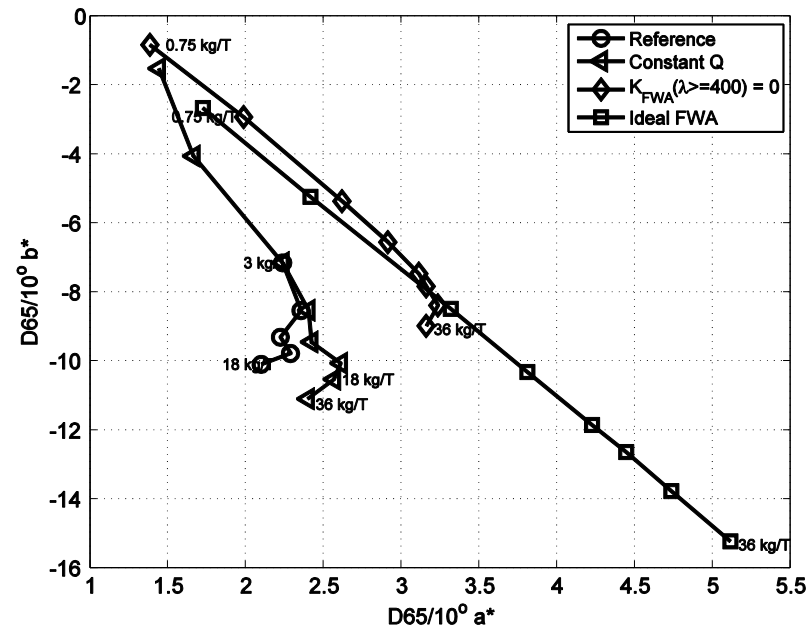

Fig 6. Effect of absorption in the emission band of the FWA on CIELAB $a^{*}$ and $b^{*}$. The measured samples (reference) show a saturation phase followed by a shift towards green (lower $a^{*}$ ) when the concentration of FWA is increased. For an ideal FWA $b^{*}$ versus $a^{*}$ is linear.

obtained up to $540 \mathrm{~nm}$ only. For the $3 \mathrm{~kg} / \mathrm{T}$ sample, the simulated $a^{*} b^{*}$ values in the case of $Q$ independent of FWA concentration (constant $Q$ ) are the same as the measured ones since this sample was used as calibration point. At higher FWA concentrations, the simulated $a^{*}$ and $b^{*}$ values are close to the measured values. The $a^{*}$ value is slightly larger for the FWA with constant $Q$ than for the FWA with varying $Q$ meaning that the samples get greener when the FWA concentration increased, and hence $Q$ shifts in the red-ward direction. However, the $a^{*}$ versus $b^{*}$ curve turns towards smaller $a^{*}$ values at higher FWA concentrations, even in the case with constant $Q$.
The simulated $b^{*}$ values for the case with a hypothetical FWA that does not absorb light at wavelengths above $400 \mathrm{~nm}$ are larger than in the case with the real FWA used in this study. The reason for this is that a significant part of the fluorescence contribution to the radiance factor occurs at excitation wavelengths between $400 \mathrm{~nm}$ and 420 nm, as shown in Fig 7. In the case where an ideal FWA is applied, $a^{*}$ and $b^{*}$ are linearly dependent and no green shift is observed within the range of FWA concentrations used in this study.

The effect on CIE whiteness of the shift of the quantum efficiency and of the absorption by FWA in the visible spectrum is shown in Fig 8 . The redward shift of the quantum efficiency has a negligible effect on the whiteness compared to the effect of the FWA absorption in the blue region of the spectrum. Fluoresced light from excitation wavelengths above $400 \mathrm{~nm}$ has a significant contribution to the whiteness. The ideal FWA, which fluoresced as much light as the real FWA without any absorption above $400 \mathrm{~nm}$ gives rise to significantly higher whiteness values.

In Fig 9, the reflectance component (obtained from $\beta_{\mathrm{R}}$ ) and the fluorescence component (total whiteness minus reflectance component) are plotted versus FWA concentration. The reflectance component decreases with increasing FWA amount because the reflected radiance factor decreases between 400 and $420 \mathrm{~nm}$ due to the absorption by the FWA. At low FWA concentration, fluorescence increases whiteness at a higher rate than the decrease in the reflected radiance factor lowers it. However, at higher FWA concentrations, the positive effect of increased fluorescence is neutralised by the decrease of the reflected radiance factor contribution to the measured whiteness.

\section{Discussion}

This study was restricted to one FWA quality added in different concentrations to the same pulp. An increase in the FWA concentration did not significantly affect the total quantum efficiency. The quantum efficiency of the FWA was shifted redward with increasing FWA concentration, but the effect on the CIE whiteness was negligible.

Light absorption by the FWA in the visible spectrum was shown to be the main cause of greening (a shift of the chromaticity towards green) and saturation of the fluorescence effect. With increasing FWA concentration, the positive effect of fluorescence is neutralised by a reduction in the reflected radiance factor in the violet-blue region of the spectrum induced by a significant absorption by the FWA in that region. Hence, the efficiency of the 


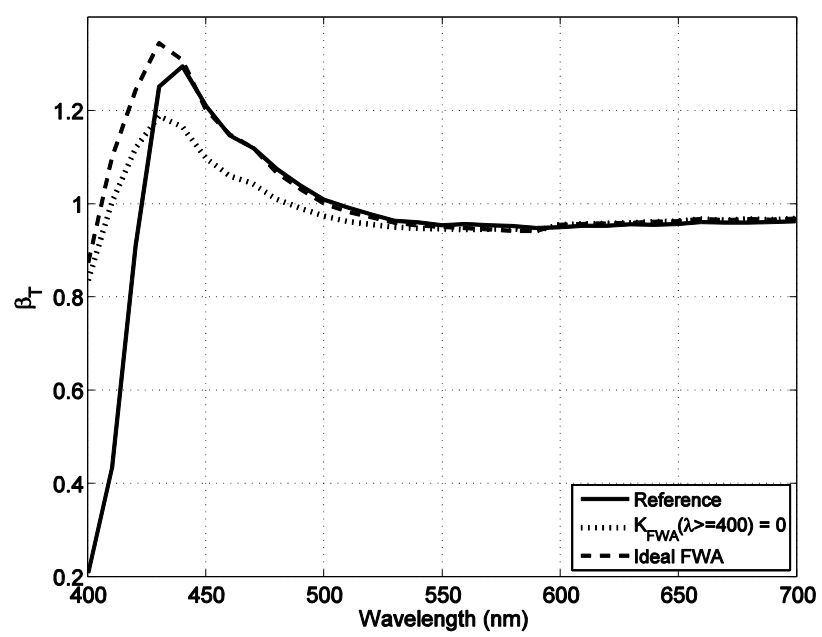

Fig 7. Effect of absorption in the emission band of the FWA on the radiance factor, illustrated with simulations for the $18 \mathrm{~kg} / \mathrm{T}$ sample. With no absorption by the FWA for wavelengths above $400 \mathrm{~nm}$, the radiance factor is higher between 400 and $420 \mathrm{~nm}$ than for the reference. However, it is lower than for the reference for wavelengths above $420 \mathrm{~nm}$. In an ideal case, the FWA would absorb and fluoresce the same amount of radiation, as the reference, but the absorption would only take place in the region below $400 \mathrm{~nm}$. A significant part of the luminescent radiance factor arises from absorption by the FWA between $400 \mathrm{~nm}$ and $420 \mathrm{~nm}$.

FWA in the uncoated papers used in this study is optically limited due to absorption by the FWA in the visible spectrum. This includes both self absorption of fluoresced light and absorption of light incident in the violet-blue part of the spectrum.

A similar effect is found for printing inks as shown by Norberg (2006). In the inkjet printing process, an increase in the ink amount added to a substrate will at a certain level no longer lead to a more saturated colour, instead this will lead to a saturation or an abrupt colour shift. The reason for the colour shift can be found in the reflectance spectrum of the printed colour. When the amount of ink exceeds a certain level, no change will occur in the absorption band. However, the transmittance will still be reduced in the pass band, thus inducing a change in the shape of the spectrum resulting in a colour shift.

This absorption of fluoresced light is well predicted by the extended K-M models, although they were derived under the assumption that the emitted wavelength was outside the absorption band of the FWA. The model does not on the other hand take into account fluorescence cascade, which is likely to occur when fluoresced light is re-absorbed in the excitation band of the FWA (Shakespeare, Shakespeare 1999). When fluoresced light is absorbed in the absorption band of the FWA, it can be further fluoresced. To account for this, Monte Carlo models will be used (Coppel et al. 2009). A prediction model would also require modelling of the absorption coefficient as a function of the FWA

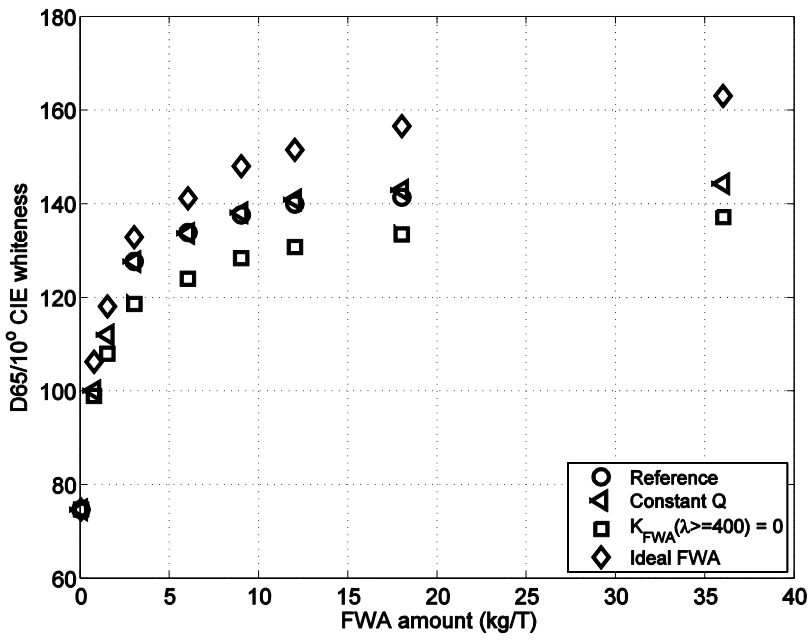

Fig 8. D65/10 $\mathrm{CIE}$ whiteness versus FWA concentration for the reference FWA (measured), and for the following cases 1) $Q$ is assumed to be constant; 2) FWA does not absorb at visible wavelengths; and 3) Ideal FWA that absorbs the same amount of radiation as the reference does but without any absorption in the visible spectrum.

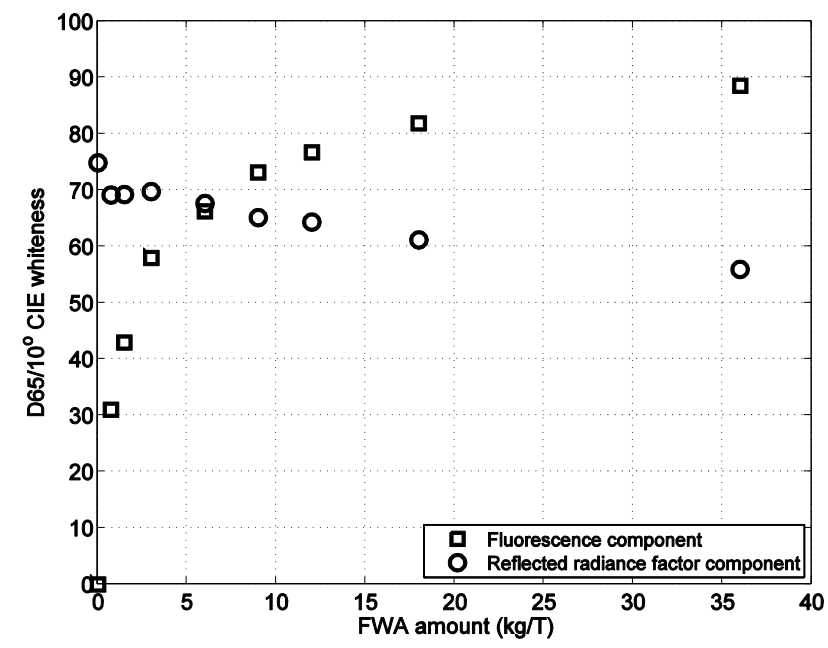

Fig 9. The contribution to whiteness from fluorescence and from reflectance plotted individually versus FWA concentration. Initially, the fluorescence component increases much faster than the reflectance component decreases. At higher FWA concentrations, the increase in fluorescence is counteracted by a decrease in the reflected radiance factor.

added to the pulp. The results were presented as function of the FWA added to the pulp prior to forming, but the calculations were based on the determined absorption coefficients. The absorption coefficient showed a non-linear dependence on the concentration of dye added to the pulp. This might be attributed to varying retention and/or a dependence of the FWA absorption properties. However, this does not affect the conclusions of this present work, since the results are based only on the actual absorption properties at each FWA concentration.

The results apply to whiteness and $L^{*} a^{*} b^{*}$ obtained with a $0^{\circ} / 45^{\circ}$ bispectrometer. On the other hand, Fig 1 shows that the radiance factor measured 
in a $\mathrm{d} / 0^{\circ}$ instrument agrees well with the radiance factor obtained with the $0^{\circ} / 45^{\circ}$ bispectrometer. The agreement might be surprising because of the difference in measurement geometry but can be explained by the calibration of the $\mathrm{d} / 0^{\circ}$ instrument that includes a geometry correction. The whiteness values obtained in both geometries are also in good agreement and the chromaticity shift occurs at the same $W_{\mathrm{CIE}}$ and $a^{*}$ value for both measurement geometries. This suggests that the conclusions of this study can be extended to whiteness measurement made with $\mathrm{d} / 0^{\circ}$ instruments.

The CIE whiteness is, according to the standard, measured on an opaque pad of samples. For these semi-infinite samples, the whiteness can be predicted from the determination of the quantum efficiency at one FWA level, assuming constant scattering. The scattering coefficient of the undyed sheet was extrapolated into the UV region of the electromagnetic spectrum. Several authors have reported a decrease in the scattering coefficient with increasing dye concentration (Koukoulas, Jordan, 1997; Rundlöf, Bristow, 1997; Shakespeare, 2000). However, a lower scattering coefficient would lead to lower absorption coefficients so that $K / S$ remains constant when determined from the reflected radiance factor of an opaque pad of samples. The determination of the scattering and absorption coefficients in regions of strong absorption is a difficult task because the reflectance of one single sheet does not significantly differ from the reflectance of an opaque pad. New methods based on angle-resolved reflectance measurements might lead to better estimation of the scattering and absorption coefficients (Neuman, Edström 2010a; 2010b)

\section{Conclusions}

The results explain why whiteness saturation and a chromaticity shift occur in uncoated papers at a certain concentration of FWA. For an opaque pad of papers, on which whiteness is measured, the whiteness at different FWA concentrations can be calculated with constant quantum efficiency and constant scattering coefficient. Higher whiteness values are obtained for larger effective FWA absorption coefficient. Because of the absorption by the FWA in the visible range of the spectrum, excessive dosage of FWA leads to a saturation of the whiteness, and a chromaticity shift towards green. The effect is predicted from the scattering and absorption coefficients of the paper without FWA and the absorption coefficient of the paper with FWA.

\section{Appendix 1: nomenclature}

$\mu$

$\lambda$

$B(\mu, \lambda)$

$Q(\mu, \lambda)$

$E(\lambda)$

$\beta_{T}(\lambda \mid E)$

$\beta_{R}(\lambda)$

$K(\lambda)$

$S(\lambda)$

$K_{\text {undyed }}(\lambda)$ Absorption coefficient of the undyed sample.

$K_{F}(\lambda)$

Excitation wavelength

Emission wavelength

Donaldson matrix.

Discrete

approximation of the bispectral radiance factor.

Quantum efficiency of the FWA molecule. Describes the energy transfer FWA.

Total quantum efficiency of the FWA at excitation wavelength $\mu$. $Q_{\text {tot }}(\mu)=\sum_{\lambda} Q(\mu, \lambda)$.

Discrete relative spectral power distribution of the illuminant.

Total radiance factor for an illuminant $E$. Sum of the reflected radiance factor, $\beta_{R}(\lambda)$ and the luminescent radiance factor $\beta_{L}(\lambda \mid E)$.

Reflected radiance factor, $\beta_{R}(\lambda) \leq 1$.

Kubelka-Munk absorption coefficient of the sample.

Kubelka-Munk scattering coefficient of the sample. the FWA. $K_{F}(\lambda)=K(\lambda)-K_{\text {undyed }}(\lambda)$. from $\mu$ to $\lambda$ upon absorption by the

\section{Acknowledgements}

The Swedish Governmental Agency for Innovation Systems (VINNOVA), the Kempe foundations, and the Knowledge Foundation (KK-stiftelsen) are gratefully acknowledged for financial support. Markku Hauta-Kasari and Jussi Parkkinen, from the University of Eastern Finland in Joensuu, are thanked for providing access to the bispectrometer and for discussions about the measurements. The authors wish to thank a reviewer for a number of valuable inputs.

\section{Literature}

Allen E. (1957): Mode of action of fluorescent whitening agents and measurement of their relative efficiency, J. Opt. Soc. Am., 47(10), 933-943.

Allen E. (1964): Fluorescent white dyes: calculation of fluorescence from reflectivity values, J. Opt. Soc. Am., 54(4), 506-514.

Bayer Chemicals (2003): Blankophor® fluorescent whitening agents - A handbook for the paper industry. 
Bristow J.A. (1994): The calibration of instruments for the measurement of paper whiteness, Col. Res. Appl., 19(6), 75483.

Bonham J.S. (1986): Fluorescence and Kubelka-Munk theory, Col. Res. Appl., 11(3), 223-230.

CIE 182:2007 (2007): Calibration methods and photoluminescent standards for total radiance factor measurements, technical report, ISBN 9783901906626.

Coppel L. G., Edström, P. and Lindquister M. (2009): Open source Monte Carlo simulation platform for particle level simulation of light scattering from generated paper structures, in Proc. Papermaking research symposium, Kuopio, Finland.

Donaldson R. (1954): Spectrophotometry of fluorescent pigments, British Journal of Applied Physics, 5, 210-214.

Fukshansky L. and Kazarinova N. (1980): Extension of the Kubelka-Munk theory of light propagation in intensively scattering materials to fluorescent media, J. Opt. Soc. Am., 70(9), 1101-1111.

Ganz E. and Pauli H.K.A. (1995): Whiteness and tint formulas of the Comission Internationale de l'Eclairage: approximations in the $L^{*} a^{*} b^{*}$ color space, Appl. Opt., 34(6), 2998-2999.

ISO 11475 (2004): Paper and board - Determination of CIE whiteness, $\mathrm{D} 65 / 10^{\circ}$ (outdoor daylight), international Organization for Standardization: Geneva, Switzerland.

ISO 11476 (2000): Paper and board - Determination of CIE whiteness, $\mathrm{C} / 2^{\circ}$ (indoor illumination conditions), international Organization for Standardization: Geneva, Switzerland.

ISO 2469 (2007): Paper, board and pulps - Measurement of diffuse reflectance factor, International Organization for Standardization: Geneva, Switzerland.

Kokhanovsky A. (2009): Radiative properties of optically thick fluorescent turbid media, J. Opt. Soc. Am. A, 26(8), 1896-1900.

Kokhanovsky A. (2010): Radiative properties of optically thick fluorescent turbid media: errata, J. Opt. Soc. Am. A, 27(9), 2084-2084.

Koukoulas, A. A. and Jordan B.D. (1997): Effect of strong absorption on the Kubelka-Munk scattering coefficient, J. Pulp Paper Sci., 24(5), 224-232.

Kubelka P. and Munk. F. (1931): Ein Beitrag zur Optik der Farbanstriche, Z. Tech. Phys., 11a, 593-601.

Kubelka, P. (1948): New contributions to the optics of intensely light-scattering materials. Part I, J. Opt. Soc. Am., 38, 448-448.

Neuman M. and Edström P. (2010a): Anisotropic reflectance from turbid media. I. Theory, J. Opt. Soc. Am., 27(5), 10321039.

Neuman M. and Edström P. (2010b): Anisotropic reflectance from turbid media. II. Measurements, J. Opt. Soc. Am., 27(5), 1040-1045.

Norberg 0. (2006): The importance of paper properties in digital colour printing, Linköping studies in science and technology - Dissertations No. 1050, Linköping University, ISBN 91-85643-66-1.

Puebla C. (2006): Whiteness assessment: a primer Concepts, determination and control of perceived whiteness, Axiphos $\mathrm{GmbH}$, Germany, revised september 2006.

Roick T. and Hunke B. (2003): New generation of FWAs (OBAs) for the paper industry, Wochenblatt für
Papierfabrikation ISSN 0043-7131, Münchner PapierSymposium (26/03/2003) 2003, 131(10), 572-575.

Rundlöf M. and Bristow J.A. (1997): A note concerning the interaction between light scattering and light absorption in the application of the Kubelka Munk equations, J. Pulp Paper Sci., 23(5), 220-223.

Shakespeare, T. and Shakespeare, J. (1999): Problems in colour measurement of fluorescent paper grades, Ana. Chim. Acta, 380, 227-242.

Shakespeare, T. (2000): Colorant Modelling for On-Line Paper Coloring: Evaluations of Mondels and an Extension to KubelkaMunk model, Phd Thesis, Tampere University of Technology, Publications 304, ISBN 952-15-0474-9.

Shakespeare, T. and Shakespeare, J. (2003): A fluorescent extension to the Kubelka-Munk model, Col. Res. Appl., 28(1), 414.

Turunen, P., Kinnunen, J, and Mutanen, J. (2010): Modeling of fluorescent color mixing by regression analysis, In Proc. CGIV 2010 - Fourth European Conference on Colour in Graphics, Imaging, and MCS/10 Vision 12th International Symposium on Multispectral Colour Science (CD-ROM), Joensuu, Finland, ISBN / ISSN: 978-0-89208-291-9, 94-100.

Uchida H. (1998): A new whiteness formula, Col. Res. Appl., 23(4), 202-209.

Wyszecki G. and Stiles, W. S. (2000): Color Science: Concepts and Methods, Quantitative Data and Formulae, 2nd Edition, pp. 968. ISBN 0-471-39918-3. Wiley-VCH.

Zwinkels J.C. Gauthier F (1999): Instrumentation, standards, and procedures used at the National Research Council of Canada for high-accuracy fluorescence measurements, Ana. Chim. Acta, 380(2-3), 193-209.

Manuscript received December 20, 2010 Accepted May 5, 2011 